\title{
Identifying Toll-Like Receptor Expression in Molluscum Contagiosum
}

\author{
Maria Yaldo ${ }^{*}$, Katherine Caretti ${ }^{2}$, Jessica Kado² and Darius R Mehregan ${ }^{2}$
}

${ }^{1}$ Wayne State University School of Medicine, USA

${ }^{2}$ Department of Dermatology, Wayne State University School of Medicine, USA

*Corresponding author: Maria Yaldo, Oakwood Dearborn Medical Park, Suite 300, 18100 Oakwood Boulevard, Dearborn, MI 48124, USA, Tel: 313-429-7843, E-mail: myaldo@med.wayne.edu

\begin{abstract}
Toll-like receptors (TLRs) are known to be involved in the immunogenic response in a variety of dermatological disorders including psoriasis, condyloma acuminata, atopic dermatitis, and cutaneous T-cell lymphoma. However, the expression of TLRs in Molluscum contagiosum has not yet been clearly defined.Expression of TLRs 1-9 was examined in molluscum by immunohistochemical staining. Results showed TLRs 2,5, and 6 stained most intensely in both the epidermis and infundibulum in molluscum cases. It was also found that there was minimal staining of TLRs 7 and 8 in molluscum cases. Condyloma acuminata cases were used as control. The nuclear factor-kappa B activation pathway was confirmed by staining for p65. All cases stained moderately for p65. The lack of expression of TLRs 7 and 8 in molluscum suggests therapeutic agonists to TLR 7 such as imiquimod may be ineffective in the treatment of molluscum. Further studies to delineate the mechanisms of viral induced immune responses will lead to the development of better anti-viral medications.
\end{abstract}

\section{Keywords}

Molluscum contagiosum, Toll-like receptor, Molluscum, TLR

\section{Introduction}

Molluscum contagiosum is a viral skin infection caused by adsDNA poxvirus, most commonly affecting children. It also occurs less frequently and may be spread as a sexually transmitted disease in adults.The infection is often self-limiting, but burdensome in immunocompetent individuals, with a mean duration of eight months. In patients with an underlying immunodeficiency, molluscum can cause an extensive and persistent infection, often causing suffering and disfigurement. The infection localizes in the epidermal layer where the virus replicates and is able to evade detection by the immune system for most of the infection period [1]. Unfortunately, there is no single stronglyeffective treatment for the management of molluscum [2]. Current interventions that do exist such as ablative therapy are painful and involve potential for scarring, hyperpigmentation, and hypopigmentation.

Toll like receptors (TLR) are pattern recognition receptors that play a vital role in the activation of both the innate and adaptive immune response by recognizing various pathogen-associated molecular patterns present in microbes to alert the immune system of suspected pathogens [3,4]. TLRs have also been shown to be significant in the initiation of the antiviral response [5]. There are at least ten human TLRs each of which are structured as single pass transmembrane receptors. It has been shown that human keratinocytes in the epidermis express TLRs [6]. TLRs 1, 2, 4, 5, 6 are all extracellular whereas TLRs 3, 7, 8, and 9 are located in the cytoplasm, hence intracellular receptors [7]. There are distinct molecular patterns on pathogens that different TLRs recognize. TLR2 and TLR 4 are known to bind to microbial components including lipopolysaccharides [8]. TLR 5 is known to recognize bacterial flagellin.TLR3 can recognize double-stranded (ds) RNA, whereas TLR7 and TLR8 bind to single-stranded (ss) RNA and are activated by imidazoquinolines. TLR9 recognizes unmethylated cysteine-phosphate-guanine (CPG)-rich motifs in DNA of bacteria and viruses [5].

Activation of TLRs triggers a strong inflammatory response and cytokine production via the nuclear factor kappa B transaction pathway [9]. This leads to production of pro-inflammatory cytokines and chemokines most notably tumor necrosis factor alpha, interleukin-12, and interferons. These activate T helper-1-dominated cellswhich play a crucial role in the elimination of pathogens including viruses [7].

There are few published reports of studies evaluating the expression of TLRs 2-4, 7, and 9 along with pro-inflammatory cytokines in molluscum specimens $[10,11]$. We investigated the expression of all TLRs 1-9 in molluscum contagiosum.

\section{Materials and Methods}

In our study, we explored TLR expression of molluscum in the epidermis and dermis by immunostaining for TLRs 1-9. Normal skin was used as a control. Condyloma was used as a comparison control group as this has been previously well characterized. Our study was approved by the Institutional Review Board of Wayne State University School of Medicine.

We retrospectively examined ten patients with a histologic diagnosis of molluscum and ten patients with a histologic diagnosis of condyloma for comparison using immunohistochemical analysis. Clinical information was gathered on all 20 patients in order to confirm the histologic diagnosis. Intensity was graded on a scale of $0-3+$ staining by two investigators for all the cases. Staining $1+$ was defined as less than $10 \%$ of keratinocytes staining, $2+$ as between $10-$

\section{Clinmed International Library}

Citation: Yaldo M, Caretti K, Kado J, Mehregan DR (2015) Identifying Toll-Like Receptor Expression in Molluscum Contagiosum. Int J Immunol Immunother 2:008

Received: February 24, 2015: Accepted: March 29, 2015: Published: March 31, 2015 Copyright: () 2015 Yaldo M. This is an open-access article distributed under the terms of the Creative Commons Attribution License, which permits unrestricted use, distribution, and reproduction in any medium, provided the original author and source are credited. 
Table 1: Condyloma Staining Results vs. Normal Skin

\begin{tabular}{|c|c|c|c|c|c|c|c|c|c|c|}
\hline Condyloma Cases & TLR 1 & TLR 2 & TLR 3 & TLR 4 & TLR 5 & TLR 6 & TLR 7 & TLR 8 & TLR 9 & NF-kB \\
\hline \multicolumn{11}{|l|}{ Case 1} \\
\hline Epidermis & - & $1+$ & - & - & $3+$ & $2+$ & - & - & - & $1+$ \\
\hline \multicolumn{11}{|l|}{ Case 2} \\
\hline Epidermis & - & $2+$ & - & - & $3+$ & $2+$ & - & - & - & $2+$ \\
\hline \multicolumn{11}{|l|}{ Case 3} \\
\hline Epidermis & - & $3+$ & - & - & $3+$ & $2+$ & - & - & - & $2+$ \\
\hline \multicolumn{11}{|l|}{ Case 4} \\
\hline Epidermis & - & $3+$ & - & $1+$ & $3+$ & $2+$ & - & - & - & $2+$ \\
\hline \multicolumn{11}{|l|}{ Case 5} \\
\hline Epidermis & - & $3+$ & - & $2+$ & $3+$ & $3+$ & - & - & - & $1+$ \\
\hline \multicolumn{11}{|l|}{ Case 6} \\
\hline Epidermis & - & $2+$ & - & - & $3+$ & $1+$ & $1+$ & - & - & - \\
\hline \multicolumn{11}{|l|}{ Case 7} \\
\hline Epidermis & - & $3+$ & - & - & $3+$ & $2+$ & - & - & - & $1+$ \\
\hline \multicolumn{11}{|l|}{ Case 8} \\
\hline Epidermis & - & $3+$ & - & $1+$ & $3+$ & $2+$ & - & - & - & $1+$ \\
\hline \multicolumn{11}{|l|}{ Case 9} \\
\hline Epidermis & - & $1+$ & $3+$ & - & $3+$ & $1+$ & - & - & - & $2+$ \\
\hline \multicolumn{11}{|l|}{ Case 10} \\
\hline Epidermis & - & $1+$ & - & $2+$ & $3+$ & $3+$ & - & - & - & $1+$ \\
\hline \multicolumn{11}{|l|}{ Normal Skin } \\
\hline Epidermis & - & $1+$ & - & $1+$ & $2+$ & $1+$ & - & - & - & - \\
\hline
\end{tabular}

Table 2: Molluscum Staining Results vs. Normal Skin

\begin{tabular}{|c|c|c|c|c|c|c|c|c|c|c|}
\hline Molluscum Cases & TLR 1 & TLR 2 & TLR 3 & TLR 4 & TLR 5 & TLR 6 & TLR 7 & TLR 8 & TLR 9 & NF-kB \\
\hline \multicolumn{11}{|l|}{ Case 1} \\
\hline Epidermis & - & $3+$ & - & $1+$ & $3+$ & $3+$ & - & $1+$ & - & - \\
\hline Infundibulum & - & $3+$ & $3+$ & $3+$ & $3+$ & $3+$ & $1+$ & $1+$ & - & $2+$ \\
\hline \multicolumn{11}{|l|}{ Case 2} \\
\hline Epidermis & - & $1+$ & - & $1+$ & $3+$ & $1+$ & - & - & - & $1+$ \\
\hline Infundibulum & - & - & $2+$ & $3+$ & $3+$ & $3+$ & $1+$ & - & - & - \\
\hline \multicolumn{11}{|l|}{ Case 3} \\
\hline Epidermis & - & $3+$ & $3+$ & $2+$ & $1+$ & $3+$ & - & $1+$ & - & $1+$ \\
\hline Infundibulum & - & $3+$ & $3+$ & $3+$ & $1+$ & $3+$ & - & $1+$ & - & $2+$ \\
\hline \multicolumn{11}{|l|}{ Case 4} \\
\hline Epidermis & - & - & $3+$ & $1+$ & $3+$ & $3+$ & - & $1+$ & - & $2+$ \\
\hline Infundibulum & - & - & $3+$ & $3+$ & $3+$ & $3+$ & $1+$ & $1+$ & - & $2+$ \\
\hline \multicolumn{11}{|l|}{ Case 5} \\
\hline Epidermis & - & $3+$ & $2+$ & $3+$ & $3+$ & $3+$ & - & $1+$ & - & $1+$ \\
\hline Infundibulum & - & $3+$ & $2+$ & $3+$ & $3+$ & $3+$ & - & $1+$ & - & $2+$ \\
\hline \multicolumn{11}{|l|}{ Case 6} \\
\hline Epidermis & - & $1+$ & $1+$ & - & $3+$ & $3+$ & - & - & - & $1+$ \\
\hline Infundibulum & - & $1+$ & $1+$ & $1+$ & $3+$ & $3+$ & $1+$ & - & - & $1+$ \\
\hline \multicolumn{11}{|l|}{ Case 7} \\
\hline Epidermis & - & $3+$ & $2+$ & - & $3+$ & $3+$ & - & $1+$ & - & - \\
\hline Infundibulum & - & $3+$ & $2+$ & $1+$ & $3+$ & $3+$ & - & $1+$ & - & $1+$ \\
\hline \multicolumn{11}{|l|}{ Case 8} \\
\hline Epidermis & - & $3+$ & - & $2+$ & $3+$ & $3+$ & - & - & - & - \\
\hline Infundibulum & - & $3+$ & - & $3+$ & $3+$ & $3+$ & - & - & - & $1+$ \\
\hline \multicolumn{11}{|l|}{ Case 9} \\
\hline Epidermis & - & $3+$ & - & $3+$ & $3+$ & $3+$ & - & $1+$ & - & $1+$ \\
\hline Infundibulum & - & $3+$ & - & $3+$ & $3+$ & $3+$ & - & $1+$ & - & $1+$ \\
\hline \multicolumn{11}{|l|}{ Case 10} \\
\hline Epidermis & - & $3+$ & - & $3+$ & $3+$ & $3+$ & - & - & - & - \\
\hline Infundibulum & - & $3+$ & - & $3+$ & $3+$ & $3+$ & - & $1+$ & - & $2+$ \\
\hline \multicolumn{11}{|l|}{ Normal skin } \\
\hline Epidermis & - & $1+$ & - & $1+$ & $2+$ & $1+$ & - & - & - & - \\
\hline Infundibulum & - & $1+$ & - & $1+$ & $2+$ & $1+$ & - & - & - & - \\
\hline
\end{tabular}

$25 \%$, and $3+$ as greater than $25 \%$ of keratinocytes staining.Location of staining was also noted, particularly whether there was staining in the epidermis or follicular infundibulum. Staining and grading techniques have been previously validated and published by the authors [12].

Applying standard protocols, 4 um formalin fixed and paraffinembedded specimens were sectioned and deparaffinized. The specimens were heated for 15 minutes in $90^{\circ} \mathrm{C}-95^{\circ} \mathrm{C}$ Citra buffer
(Dako; Carpenteria CA), pH6, to retrieve antigens. Endogenous peroxidase activity was blocked with hydrogen peroxide for ten minutes. Specimens were incubated in primary TLR antibodies, TLRs 1-9, for 3 hours at 1:100 dilution (Santa Cruz Biotechnology, Santa Cruz, CA). (Table 1,2) All antibodies are polyclonal, except for the anti-TLR9 which was monoclonal, and all are directed to a portion of human TLR protein. Antigen detection was performed using Dako Envision Flex detection kit with 3-amino-9ethylcarbazole chromogen 


\section{TLR 1}

\section{TLR 2}

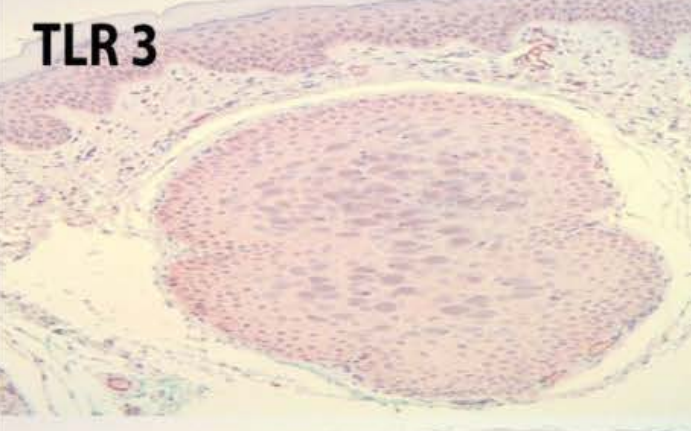

\section{TLR 4}

TLR 5

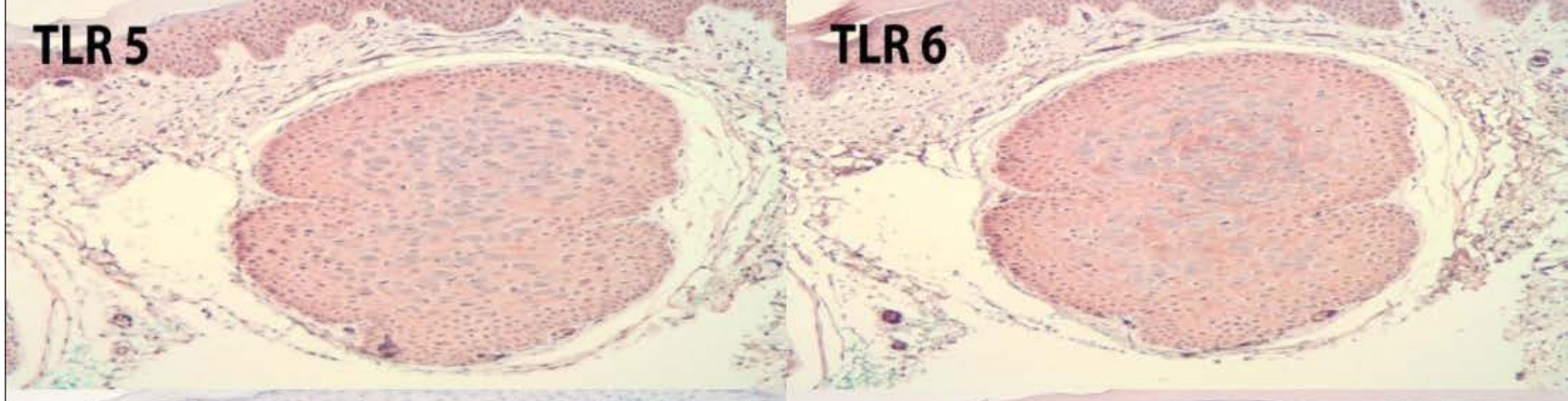

\section{TLR 7}

\section{TLR 9}

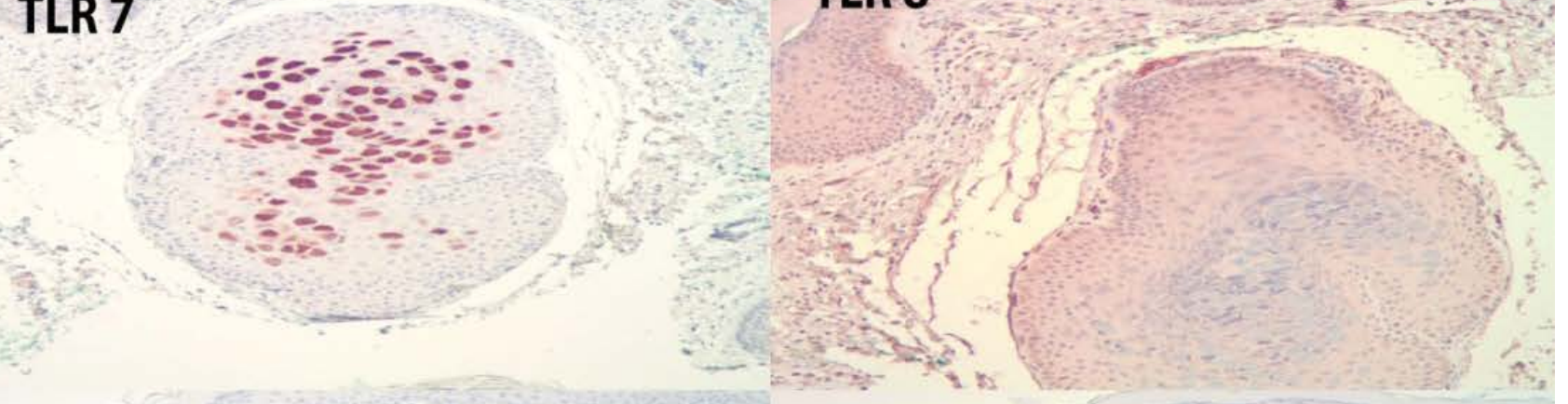

\section{TLR 8}
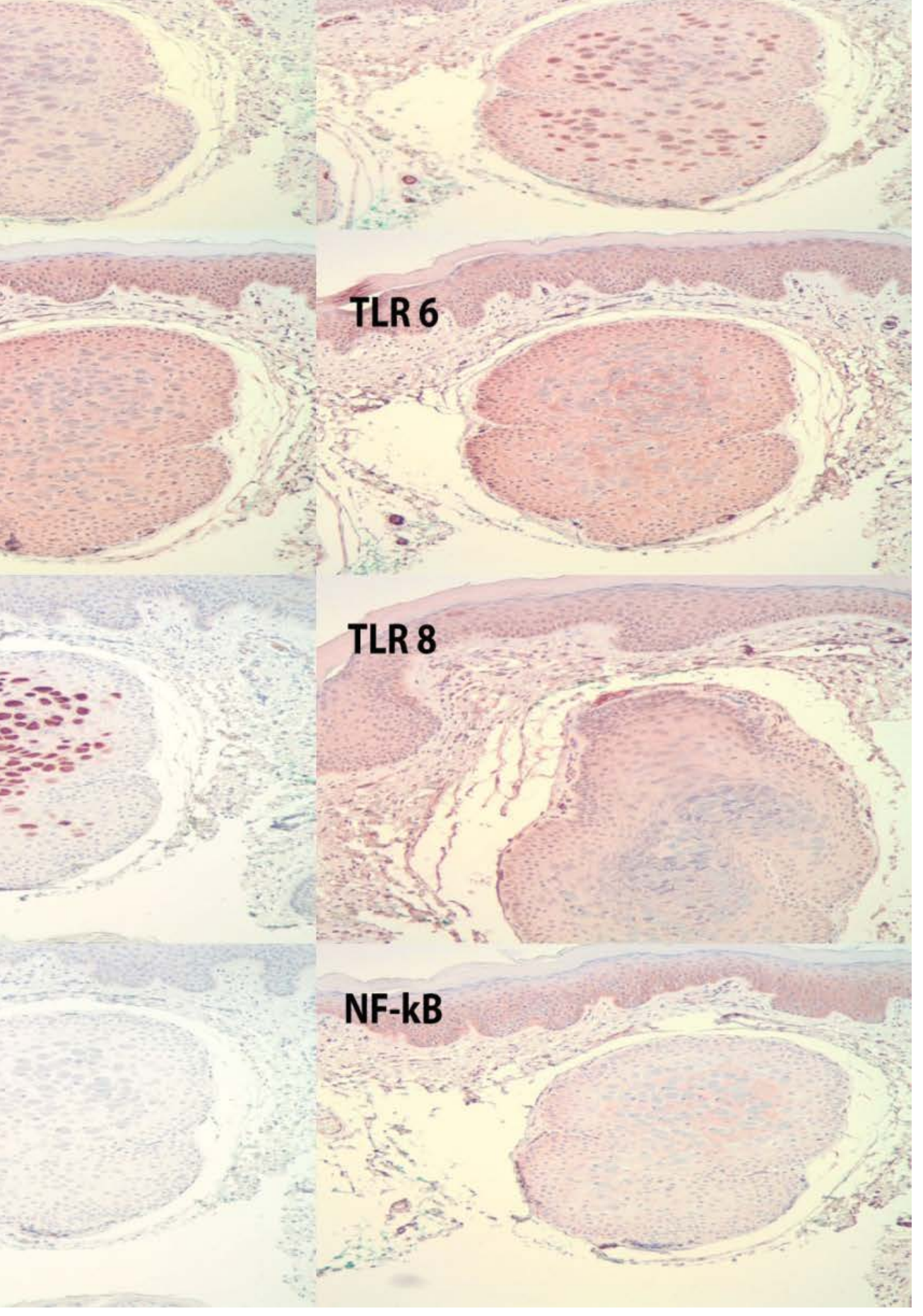

Figure 1: Staining patterns of TLR 1-9 and NF-Kb in a case of molluscum contagiosum, showing increased expression of TLR's 2,3,4,5,6 and 8. There is focal staining of molluscum bodies in the follicular infundibulum. NF-Kb is also expressed. Hematoxylin counterstain; $4 x$ objective. 
Table 3: Toll Receptor antibody clones utilized

\begin{tabular}{|l|l|}
\hline Toll Receptor antibody clones utilized & \\
\hline TLR1 (B-23) & sc-130896 \\
\hline TLR2 (H-175) & sc-10739 \\
\hline TLR3 (H-125) & sc 10740 \\
\hline TLR4 (H-80) & sc 10741 \\
\hline TLR5 (H-127) & sc-10742 \\
\hline TLR6 (H-90) & sc-30001 \\
\hline TLR7 (H-114) & sc-3004 \\
\hline TLR8 (H-114) & sc-25467 \\
\hline TLR9 (26C593) & sc-52966 \\
\hline
\end{tabular}

on a Dako Link 48 automated immunostainer (Dako) (Table 3).

\section{Results}

\section{TLR 1-9 molluscum contagiosum}

Henderson Patterson bodies of molluscum contagiosum were present within the follicular infundibulum of all 10 specimens. There was no significant staining noted for TLR 1 and 9. TLR 3 and TLR 4 were up-regulated and stained $2-3+$ in $70 \%$ of the cases with staining in both the epidermis and infundibulum. TLR 3 also stainedendothelial cells while TLR 4 staining was predominantly more intense in the infundibulum. TLRs 2, 5, 6 stained most intensely at a $3+$ in both the epidermis and infundibulum. There wasminimal staining at $1+$, mostly in the infundibulum, in $40 \%$ of the cases for TLR7 with the remaining cases showing no stain. TLR8 showed focal stainingin the majority of cases at a 1+ in both the epidermis and infundibulum.

\section{TLR 1-9 condyloma acuminata}

There was no significant staining noted for TLRs $1,3,8$, and 9 . TLR 7 exhibited minimal staining at $1+$ in $20 \%$ of the cases notably in the granular cell layer of the epidermis. TLR 4 showed slight staining at $1-2+$ in only $40 \%$ of cases. TLR 5 stained most intensely at $3+$ with all the cases whereas TLRs 2 and 6 moderately stained with $75 \%$ of cases staining at a 2-3+. The majority of staining of TLR 6 was noted in the granular cell layer of the epidermis.

\section{P65}

P65 was measured to confirm the NF-kappa B activation pathway induced by the TLRs studied. There was 1-2+ staining for $\mathrm{p} 65$ in both molluscum and condyloma samples.

\section{Discussion}

This is the first attempt to try and define the expression of TLRs 1-9 in molluscum using immunohistochemistry in the epidermis and dermis. All of the cases we studied had a positive skin biopsy for molluscum. Our results indicated that TLRs 2, 5, and 6 stained most intensely in both the epidermis and infundibulum. Similarly, condyloma staining was also intense for TLR 5 along with moderate staining for TLRs 2 and 6. TLRs2, 5, and 6 are all extracellular receptors that recognize microbial components [7]. Further research is needed to better elucidate viral activation of toll-like receptors.

Previous reports have also investigated the expression of TLRs in molluscum tissue using immunohistochemistry $[10,11]$. Ku et al. looked at the expression of 5 TLRs $(2,3,4,7,9)$ in molluscum and verruca vulgaris and found that compared to normal skin, TLRs 3 and 9 were up-regulated in both conditions. Our study also showed an increased expression of TLR 3 in molluscum, however no upregulation for TLR 9 compared to no significant expression of both TLRs in condyloma. TLR 3 is a known intracellular receptor that can recognize dsRNA and non-coding RNA from necrotic keratinocytes providing a trigger for skin barrier repair [13]. Because molluscum is caused by a dsDNA poxvirus, the up-regulation of TLR 3 suggests a secondary process induced by the disruption of keratinocytes by the molluscum virus.

Bakry et al. specifically studied the immunolocalization of TLR2 in molluscum and found it to be up-regulated compared to normal skin with a predominantly membranous and nuclear pattern of staining
[10]. This is analogous to our study where we found TLR 2 to be upregulated in molluscum compared to condyloma. TLR 2 is known to recognize microbial components notably lipopolysaccharides of bacteria; however our study suggests a virally induced mechanism of TLR 2 up-regulation specifically by components of the molluscum virus.

In addition to looking at TLRs 1-9 in molluscum, we also investigated TLRs in condyloma as a control group. We noted upregulation of TLRs 2, 5, and 6, but no significant expression of TLRs 1, 3, 4 and 7-9. Shi et al. also found increased expression of TLR 2 along with 3, 4, 7-9, and Myd88 in condyloma, however other studies evaluating TLRs in condyloma are rare [14] (Figure 1).

Imiquimod is known to act upon TLR 7 and/or TLR 8 exerting agonistic effects inducing expression of proinflammatory cytokines. Few studies have been done evaluating the effectiveness of imiquimod for treating molluscum [15-17]. Theos et al. performed a double-blind, randomized pilot trial invoking only 23 children and found imiquimod to be effective compared to vehicle cream with $33 \%$ children exhibiting complete clearance as opposed to $9 \%$ children receiving the vehicle cream after 12 weeks of treatment [15]. However, two larger randomized, vehicle-controlled, doubleblind trials revealed that imiquimod was not effective in treatment of molluscum in children [18]. The trials involved a combination of 702 pediatric subjects with molluscum aged 2-12 years in which 470 of the participants were treated with imiquimod $5 \%$ cream 3 times per week. At week 18, complete clearance of molluscum lesions was assessed and it was found that imiquimod was no more effective than vehicle containing cream in the treatment of molluscum $(24 \%$ vs. $26 \%$ in one trial, $24 \%$ vs. $28 \%$ in the other) [19]. One reason may be due insufficient penetration of imiquimod to reach the molluscum virus which embeds deeply into the epidermis and follicular infundibulum. It may also be due to minimal expression of agonistic receptors TLR7 and 8 in molluscum infected tissues. Our study in fact did not show significant up regulation of TLR 7 and 8 with only $40 \%$ of cases staining at a $1+$ for molluscum. This may suggest that due to the relative lack of expression of TLR 7, imiquimod may not be effective for the treatment of molluscum contagiosum. Also, as the viral bodies were primarily observed within follicular infundibula, there may be insufficient penetration of topically applied imiquimod to stimulate an inflammatory response.

The results of this study should be used to further delineate the mechanisms of viral induced immune responses and hopefully help to develop better anti-viral medications.

\section{References}

1. Chen X, Anstey AV, Bugert JJ (2013) Molluscum contagiosum virus infection. Lancet Infect Dis 13: 877-888.

2. van der Wouden JC, van der Sande R, van Suijlekom-Smit LW, Berger M, Butler CC, et al. (2009) Interventions for cutaneous molluscum contagiosum. Cochrane Database Syst Rev: 4.

3. Medzhitov R (2001) Toll-like receptors and innate immunity. Nat Rev Immuno 1: $135-145$.

4. Iwasaki A, Medzhitov R (2004) Toll-like receptor control of the adaptive immune responses. Nat Immunol 5: 987-995.

5. Kawamura T, Ogawa Y, Aoki R, Shimada S (2014) Innate and intrinsic antiviral immunity in skin. J Dermatol Sci 75: 159-166.

6. Miller LS, Modlin RL (2007) Toll-like receptors in the skin. Semin Immunopathol 29: 15-26.

7. Mempel M, Kalali BN, Ollert M, Ring J (2007) Toll-like receptors in dermatology. Dermatol Clin 25: 531-540, viii.

8. Pivarcsi A, Bodai L, Réthi B, Kenderessy-Szabó A, Koreck A, et al. (2003) Expression and function of Toll-like receptors 2 and 4 in human keratinocytes. Int Immunol 15: 721-730.

9. Li ZJ1, Sohn KC, Choi DK, Shi G, Hong D, et al. (2013) Roles of TLR7 in activation of NF-îم B signaling of keratinocytes by imiquimod. PLoS One 8: e77159.

10. Bakry OA, Abdou AG, Gewale H, Seleit I (2013) Immunolocalization of toll-like receptor 2 in viral warts and molluscum contagiosum. Anal Quant Cytopathol Histpathol 35: 316-323. 
11. Ku JK, Kwon HJ, Kim MY, Kang H, Song PI, et al. (2008) Expression of Tolllike receptors in verruca and molluscum contagiosum. J Korean Med Sci 23 : 307-314

12. Huizenga T, Kado J, Mehregan DR, Diamond S (2015) Identifying Toll-like receptor expression in cutaneous sarcoidosis. Am J Dermatopathol 37: 6772.

13. Borkowski AW, Kuo IH2, Bernard JJ3, Yoshida T4, Williams MR1, et al (2015) Toll-like receptor 3 activation is required for normal skin barrier repair following UV damage. J Invest Dermatol 135: 569-578.

14. Shi YJ, Yang J, Yang W (2013) Mechanistic investigation of immunosuppression in patients with condyloma acuminata. Mol Med Rep 8: $480-486$
15. Theos AU, Cummins R, Silverberg NB, Paller AS (2004) Effectiveness of imiquimod cream $5 \%$ for treating childhood molluscum contagiosum in a double-blind, randomized pilot trial. Cutis 74: 134-138, 141-2.

16. Gamble RG, Echols KF, Dellavalle RP (2012) Imiquimod vs cryotherapy for molluscum contagiosum: a randomized controlled trial. Arch Dermatol 148: 109-112.

17. Al-Mutairi N, Al-Doukhi A, Al-Farag S, Al-Haddad A (2010) Comparative study on the efficacy, safety, and acceptability of imiquimod $5 \%$ cream versus cryotherapy for molluscum contagiosum in children. Pediatr Dermatol 27: 388-394.

18. Katz KA1 (2014) Imiquimod is not an effective drug for molluscum contagiosum. Lancet Infect Dis 14: 372-373.

19. DailyMed (2014) Aldara (imiquimod) cream for topical use. 